Mediterránea Ser. Biol. (1987), n. o 9. Pág. 29-40

\title{
AVES NIDIFICANTES EN HUECOS DE LOS NARANJOS
}

\author{
por \\ J. A. GIL-DELGADO y E. BARBA
}

\section{RESUMEN}

Dos especies de avés crían en los huecos de los naranjos, el Carbonero Común y el Torcecuello. El Carbonero Común presenta densidades que se insertan en el intervalo de los bosques caducifolios. La población se caracteriza por presentar parejas que no crían. La supervivencia en el nido es similar al de otras especies que construyen nidos abiertos, por lo que criar en huecos en el naranjal, no ofrece ventajas en el éxito reproductor. La predación ejerce una fuerte presión durante el período nidícola. La supervivencia de los pollos en el nido está determinada por la «calidad» del hueco, siendo la altura a la que está situada la entrada uno de los factores influyentes. Con estas características, la mortalidad una vez abandonado el nido debe ser baja para mantener la población estabilizada.

\section{SUMMARY}

Parus major and Jyns torquilla breed in natural holes in orange groves. $P$. major has a density of 5-8 pairs $/ 10$ ha. and $J$. torquilla has a density of $0.6-1.2$ pairs/10 ha. Clutch-size of Great Tit is 6.4 eggs $(n=10)$. There are some Great Tit pairs which do not bred, although these pairs keep a territory throughout the breeding season. The breeding success is similar to open-nesting species, so nesting in orantge tree-holes has not advantage for this species. Predations is the most important cause of nest mortality. "Quality» of tree-holes must be important in nest survival. Mortality after leaving the nest must be low to keep a stable population.

\section{INTRODUCCION}

La presencia del Carbonero Común (Parus major) como especie nidificante en los naranajes, es señalada por GIL-DELGADO (1983), al mostrar el

(1) Departamento de Ecología, Universidad de Valencia. Burjassot (Valencia). 
estatus poblacional durante la temporada reproductora en el período de 19751980. Sin embargo, aunque la información sobre otras especies que crían en este hábitat es notable (véase p. e. GIL-DELGADO y ESCARRE 1977, GILDELGADO 1981, ESCOBAR y GIL-DELGADO 1984), el conocimiento sobre la biología del Carbonero Común durante la estación reproductora es escaso, en parte porque sus hábitos de nidificación hacen más laboriosa la detección de sus nidos.

El Carbonero Común es una especie bien conocida, por la facilidad con que acepta las cajas anidaderas. No obstante, la documentación relacionada con la nidificación en ausencia de cajas es reducida (véase p. e. NILSSON 1975). La necesidad de trabajos de este tipo, manifestada por diversos autores (KREBS 1971, DUNN 1977, PERRINS 1979, entre otros), nos anima a ofrecer los resultados obtenidos hasta el momento en torno al tamaño de la población, el éxito reproductor y la influencia de los huecos en la supervivencia, al señalar NICE (1957) que las aves que establecen sus nidos en huecos tienen un éxito reproductor superior al de especies que crían en nidos abiertos.

La segunda especie, el Torcecuello (Jynx torquilla), reside en la parcela estudiada desde 1981, comprobándose su nidificación en la misma en 1985.

\section{AREA DE ESTUDIO}

El área de estudio está constituida por una parcela de $16.919 \mathrm{Ha}$., situada en el término municipal de Sagunto (Valencia). Las características de los huertos, y la composición del estrato herbáceo, pueden consultarse en GILDELGADO y ESCARRE (1977) y GIL-DELGADO et al. (1979). No obstante, debemos señalar que, desde 1976, año en que la totalidad de la parcela estaba cubierta de arbolado, la superficie ocupada por los naranjos se ha reducido, por actuaciones relacionadas con la renovación de los huertos. En la figura 1 puede observarse que el proceso de renovación de los huertos sigue vigente.

\section{MATERIAL Y METODO}

Para determinar el número de parejas que residen en el área de estudio hemos empleado la técnica de la parcela (BLONDEL 1965, GARCIA y PURROY 1973), y el de la búsqueda de nidos (SMITH 1943, VAL NOLAN 1963), método mixto que se viene utilizando en este escenario desde 1975 (GILDELGADO 1983). La distribución uniforme de los naranjos facilita la representación de todos ellos sobre los planos de trabajo confeccionados.

En 1985 se revisaron $12 \mathrm{Ha}$. al menos dos veces durante la estación de nidificación. En aquellos lugares donde conocíamos la existencia de una pareja cuyo nido no había sido localizado, se intensificó la búsqueda. Así, aunque sobre la superficie revisada se localizaron todos los nidos, éstos se encontraron en distintas fases de actividad. Los nidos localizados se visitaban dos veces por semana.

El trabajo desarrollado en 1985, nos permitió cartografiar la totalidad de los huecos existentes en la parcela, de manera que, en 1986, todos los nidos se descubrieron en fase de construcción o durante la deposición de los huevos. Todos los huecos eran visitados semanalmente, siendo más asidua la visita a los nidos localizados. 


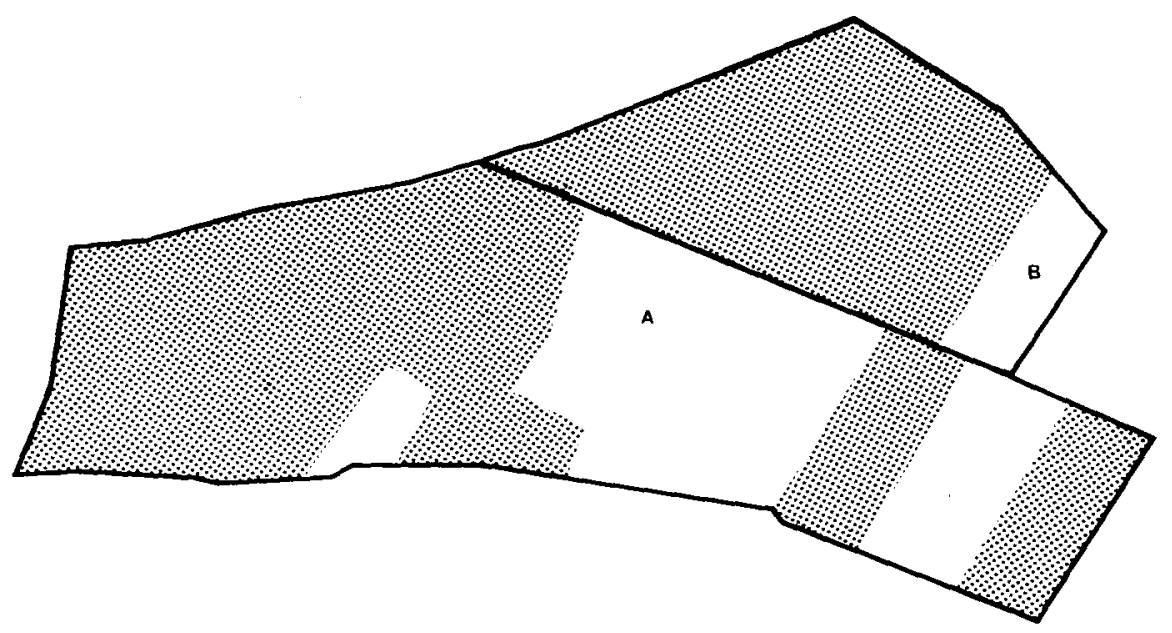

$100 \mathrm{~m}$.

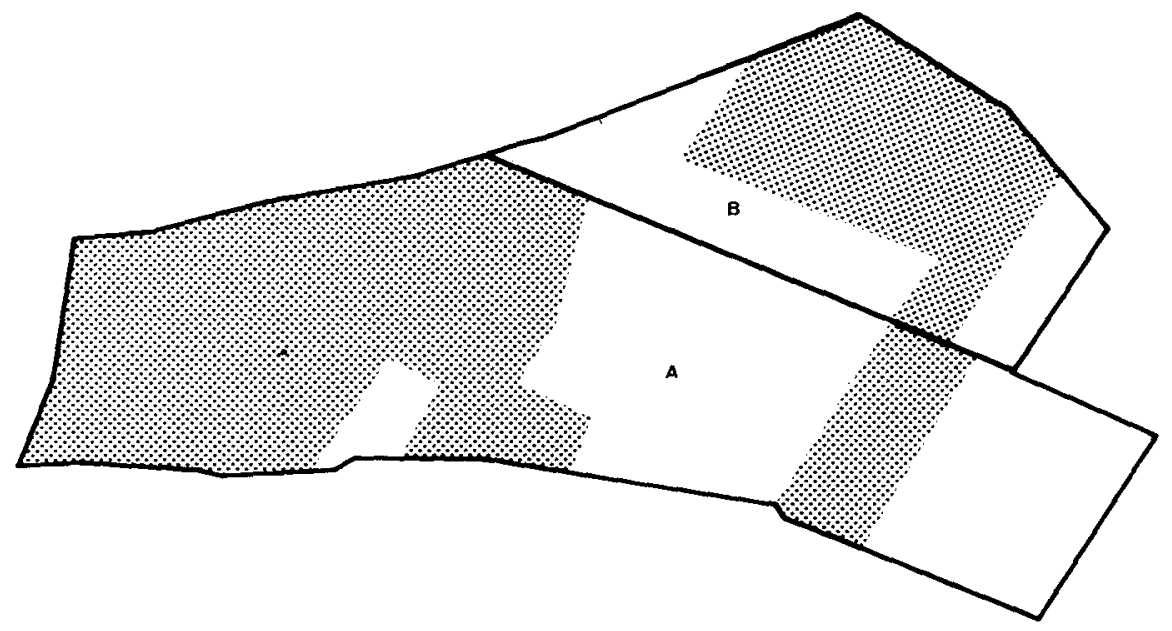

Figura 1.-Distribución de la superfície arbolada (zona punteada), en la parcela 1A) 1985. 1B) 1986. Se indican los sectores A y B.

La parcela de la figura 1 está dividida en dos sectores. En 1985 los dos sectores contenían cajas anidaderas, que fueron retiradas del sector A en 1986, con la excepción de una, por razones ajenas. Todas las cajas se colocaron en 1986 en el sector B. Por estar la parcela en estudio desde 1975, el apartado correspondiente a la densidad recoge la totalidad de parejas en el área de estudio desde esa fecha. En los años 1978 y 1979 no se censó la población de Carbonero Común. 
Los resultados que guardan relación con el resto de los temas tratados proceden exclusivamente de los nidos localizados en huecos. La mayoría pertenecen a las temporadas reproductoras de 1985 y 1986, pero incluimos tres con procedencia anterior.

Para visualizar el interior de los nidos, utilizamos una bombilla de $3.5 \mathrm{w}$., conectada mediante un cable semirrígido a una batería de 4.5 v. Este sistema nos permitió observar el interior de los huecos.

\section{RESULTADOS}

\section{Densidad del Carbonero Común}

La densidad del Carbonero Común en la parcela oscila entre 5 y 8 parejas por $10 \mathrm{Ha}$. (GIL-DELGADO 1983). Las densidades en 1985 y 1986 fueron de 7.7 y 8.3 parejas por $10 \mathrm{Ha}$; ; estos valores no difieren de las densidades de temporadas anteriores. El efecto de las cajas en 1985 debe despreciarse, pues éstas se colocaron una vez que los territorios de las distintas parejas ya se conocían. En la figura 2 se puede observar la variación de la densidad desde 1975.

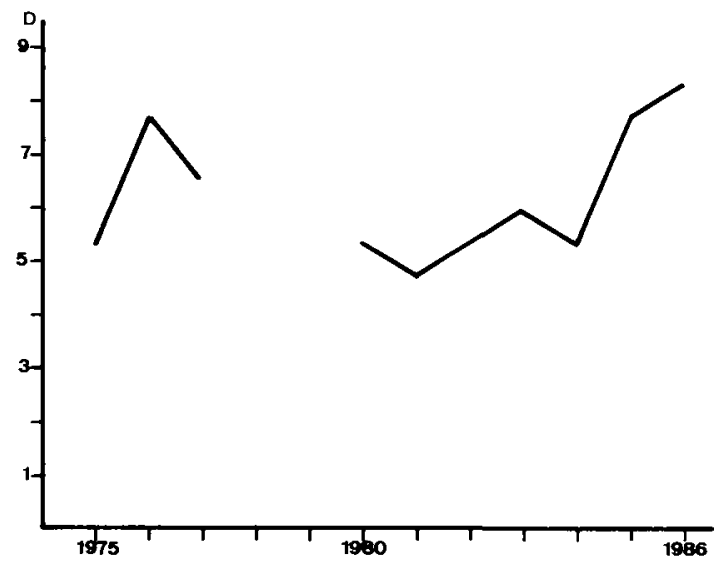

Figura 2.-Evolución de la población de Parus major en la parcela durante período de 1975 a 1986. $\mathrm{D}=$ densidad, expresada en número de parejas/10 Ha. Los valores de 1975 a 1980 proceden de GIL-DELGADO (1983).

Durante las dos primeras temporadas de estudio (1975 y 1976), la parcela estaba cubierta de arbolado en su totalidad. Desde entonces, el arbolado ha sufrido una reducción paulatina, quedando en la actualidad circunscrito a las zonas que muestra la figura 1B. No obstante, esta reducción del arbolado no ha ocasionado el descenso de parejas asentadas en la parcela, aunque sí ha incidido sobre su distribución en la zona, al concentrarse en las zonas arboladas. Este proceso se puede observar en la figura 3, donde se presenta la distribución de las parejas en las temporadas de nidificación correspondientes a los años 1976, 1981, 1985 y 1986. 

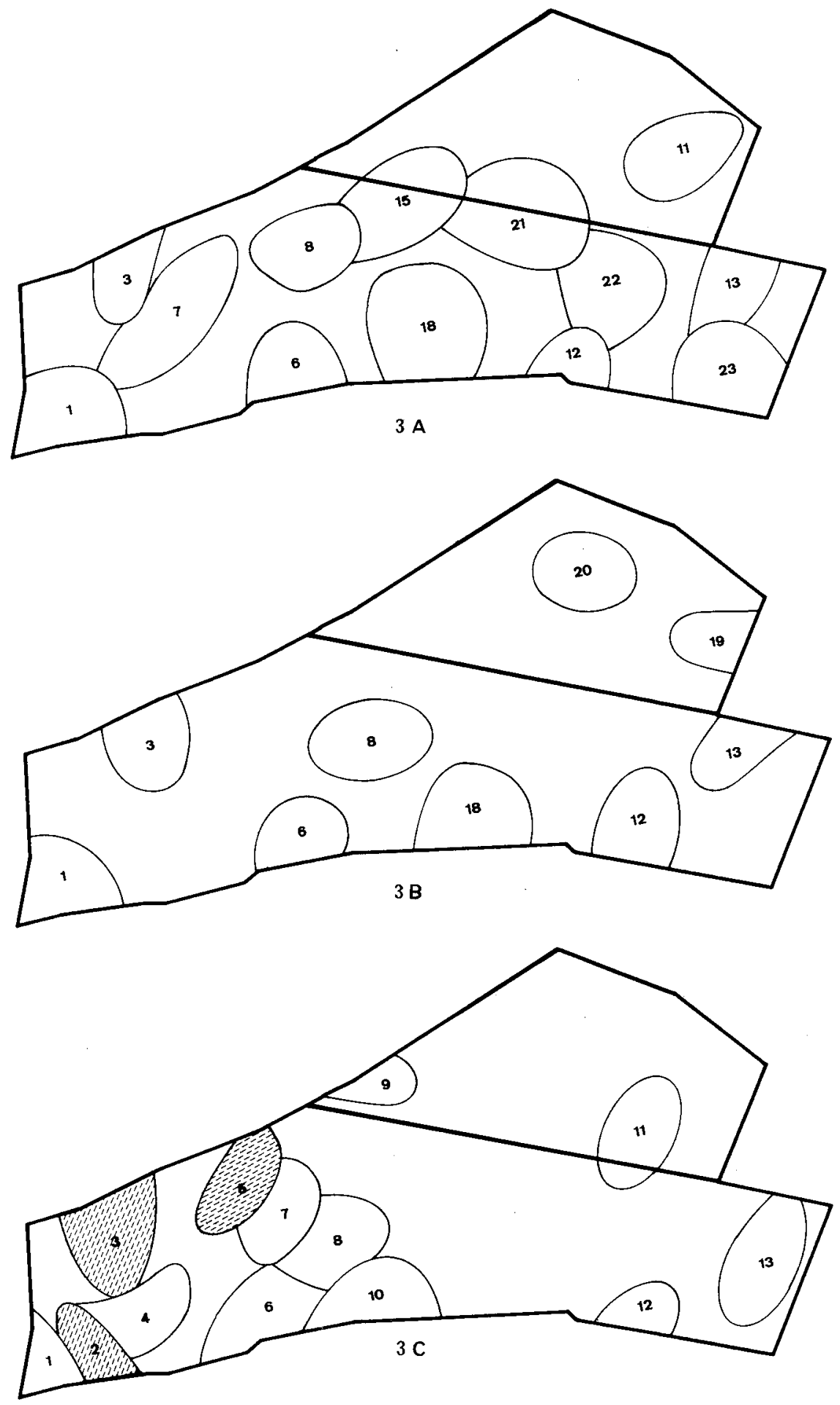


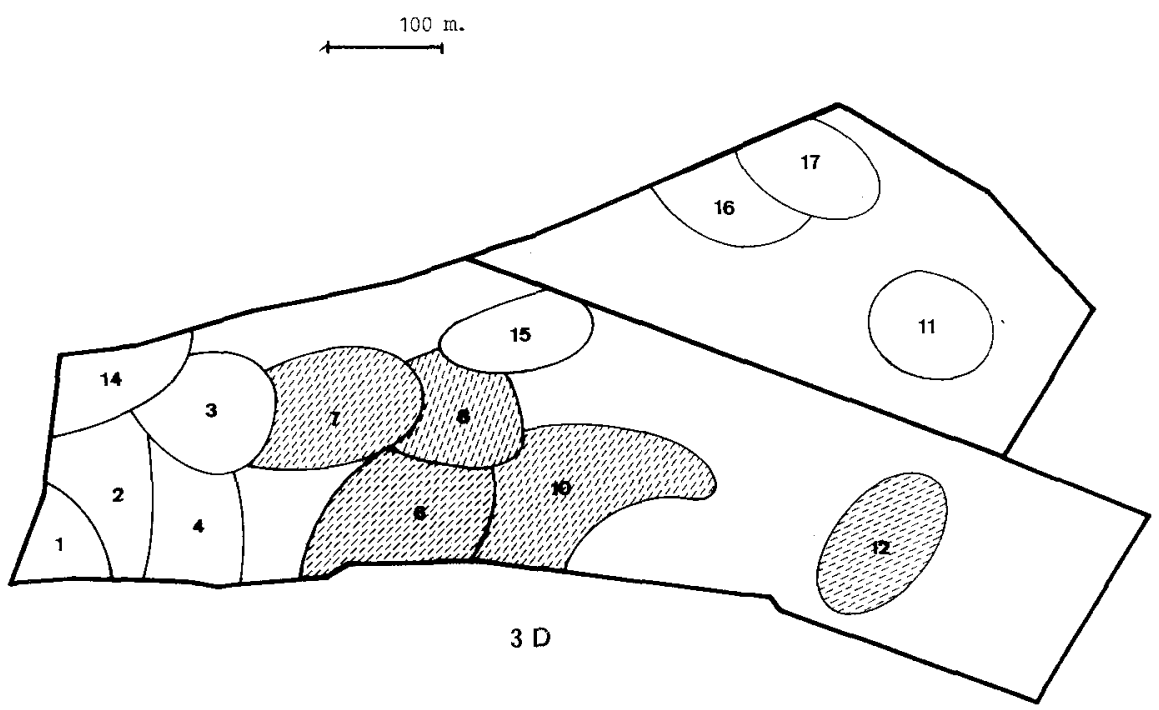

Figura 3.-Distribución de las parejas de $P$. major en los años 1976 (Fig. 3A), 1981 (Fig. 3B), 1985 (Fig. 3C) y 1986 (Fig. 3D). Los territorios sombreados en las figuras 3C y 3D corresponden a las parejas de las que se tienen sospechas fundadas de no intentar la nidificación.

\section{Parejas que no crían}

En 1985, de las 13 parejas que residían en la parcela, cuatro situaron el nido en cajas. De las nueve restantes, 5 ocuparon huecos de los naranjos. De las parejas $2,3,5$ y 6 (figura $3 \mathrm{C}$ ) no existe constancia de que intentaran criar. Las tres primeras se detectaban contínuamente sobre la parcela. La pareja 6 tenía parte de su territorio en el exterior de la parcela. Además, la pareja 9 de la misma figura, conocida desde primeros de marzo, no intentó criar hasta que tuvo un nidal disponible. La caja se colocó el 20 de abril y al día siguiente fue ocupada por la pareja propietaria del territorio.

En 1986, tras quitar los nidales del sector A, nos encontramos con el cuadro que refleja la figura 3D. Cinco parejas de este sector no criaron. Como en 1985, estas parejas eran detectadas contínuamente en sus territorios. Por otra parte, el mejor conocimiento de los huecos y la visita semanal a los mismos, hacen más improbable no descubrir los nidos en caso de que hubieran existido.

\section{Situación de los nidos}

Los nidos de Carbonero Común se disponen sobre el tronco principal y, en general, a escasa altura $(14-40 \mathrm{~cm} ., \mathrm{n}=10)$ con la abertura situada en la parte superior $(n=4)$ o lateralmente $(n=6)$. Tres nidos tenían la abertura entre 60-100 cm. El diámetro de la abertura de entrada oscila entre 3 y $13 \mathrm{~cm}$. y la profundidad de la cavidad entre 0 y $39 \mathrm{~cm}$. Todos los huecos proceden de la putrefacción del tronco. El diámetro mínimo de un tronco ocupado es 
de $26 \mathrm{~cm}$., por lo que huecos situados en árboles más jóvenes parecen ser insuficientes para las necesidades de la especie.

Los tres nidos conocidos de Torcecuello estaban ubicados en el mismo hueco. El diámetro de entrada del mismo era de $4.5 \mathrm{~cm}$., la profundidad de 39 $\mathrm{cm}$. y la entrada era lateral y estaba situada a $79 \mathrm{~cm}$. del suelo. Este hueco también lo utilizó el Carbonero Común en 1986 (véase tabla III).

\section{Tamaño de la puesta y éxito reproductor del Carbonero Común}

Sobre 10 puestas conociddas, el tamaño medio de la puesta es de .4 huevos por nido, con una distribución de dos puestas de 5 huevos, tres de 6 , cuatro de 7 y una de 8 .

La tabla I muestra el éxito de la eclosión y la supervivencia en función del número inicial de huevos. Los 67 huevos se corresponden con 11 nidos (uno más que para obtener el tamaño medio de la puesta y fue abandonado antes de que la puesta estuviera completa). Otros dos nidos se encontraron durate el período de deposición, o en los primeros días del período de incubación, pero no se pudo precisar el número de huevos. Con estos, son 13 el número de nidos, de los cuales cinco $(38.6 \%)$ dejan pollos volanderos. De cinco nidos en los que conocemos al predador, la Culebra Bastarda (Malpolon monspessulanus) devoró tres nidades, y la Comadreja (Mustela nivalis) dos.

\section{TABLA I}

Éxito en la eclosión y en la superviviencia. El número de huevos de la primera fila se corresponde con once nidos. En la segunda fila incluimos dos nidos en los que no pudo precisarse el número de huevos

\begin{tabular}{cccccc} 
N. ${ }^{\circ}$ NIDOS & $\mathbf{N}^{\circ}{ }^{\circ}$ HUEVOS & ECLOSIONES & $\%$ & SUPERVIVIENTES & $\%$ \\
\hline \multirow{2}{*}{13} & 67 & 44 & 65,6 & 19 & 28,3 \\
& & 9 & 69,2 & 5 & 38,5
\end{tabular}

\section{TABLA II}

Pollos que consiguen volar y muertos en función de la altura (h) a la que estaba situada la abertura de entrada al nido.

Los pollos pertenecen a 13 nidos.

POLLOS MUERTOS POLLOS QUE VUELAN

\begin{tabular}{lrrr}
\hline Nidos $(\mathrm{h}=60-100 \mathrm{~cm})$. & 5 & 10 & 15 \\
Nidos $(\mathrm{h}=0-40 \mathrm{~cm})$. & 29 & 9 & 38 \\
\hline & 34 & 19 & 53
\end{tabular}




\section{TABLA III}

Fenología de las cuatro nidadas criadas en el mismo hueco

\begin{tabular}{|c|c|c|c|c|c|c|}
\hline AÑ 0 & PUESTA & 1.er HUEVO & EMANCIPACIÓN & TAMAÑO DE LA PUESTA & SUPERVIVIENTES & ESPECIE \\
\hline 1985 & $1 .^{a}$ & 21-24-IV & $27-\mathrm{V}$ & $?$ & 5 & Torcecuellos \\
\hline 1985 & $2 .^{a}$ & 4-VI & 9-VII & 7 & 5 & Torcecuellos \\
\hline 1986 & $1 .^{a}$ & $5-\mathrm{VI}$ & $13-$ VII & 11 & 4 & Torcecuellos \\
\hline 1986 & 1.1 & $17-I V$ & $30-V$ & 8 & 5 & Carbonero \\
\hline
\end{tabular}

Al considerar la supervivencia en función del número de pollos eclosionados y en relación con la altura a que está situada la entrada del nido, los nidos con abertura situada entre los $60-100 \mathrm{~cm}$. presentan un éxito reproductor mayor $\left(\mathrm{Z}^{2}=8.5, \mathrm{p}<0.01\right.$, valor obtenido de la tabla II $)$.

\section{El Torcecuello}

Esta especie está asentada en la parcela desde 1981, año en que aparece la primera pareja. En 1985, una segunda pareja se establece en el área. La figura 4 permite apreciar la distribución de las dos parejas en la parcela. La pareja asentada en 1985 hace dos puestas en este año y una en 1986. La tabla III presenta el desarrollo de estas nidadas, junto con una de Carbonero Común, procedente de 1986, que utilizó para ubicar el nido este mismo hueco.

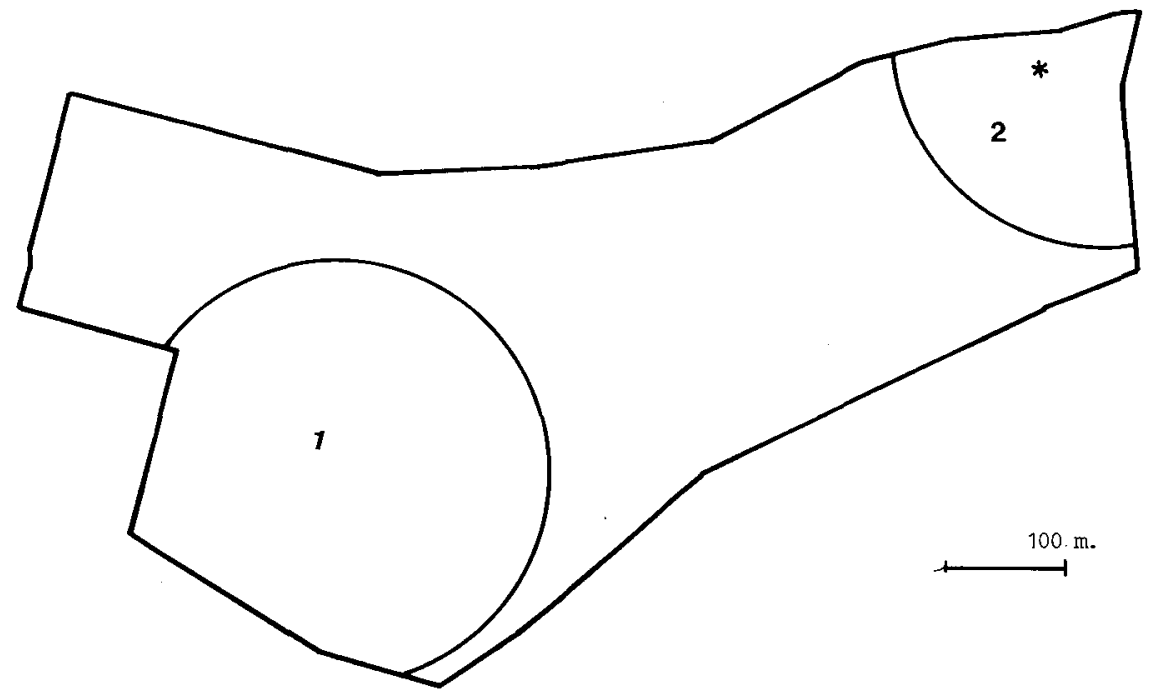

Figura 4.-Distribución de las dos parejas de Jynx torquilla en el área de estudio. La pareja 1 procede de 1981, y la pareja 2 de 1985 . La estrella, señala la situación del nido utilizado por $J$. torquilla en 1985 y 1986, y por $P$. major del territorio 2 de la figura 3 en 1986. 


\section{DISCUSION}

En ausencia de cajas,la densidad del Carbonero Común varía según el tipo de hábitat. En los bosques de coníferas, las densidades oscilan entre $0 \mathrm{y}$ 2.5 parejas por $10 \mathrm{Ha}$. (VAN BALEN 1973, PEDROCHI 1973, 1975). HERRERA (1978) señala densidades similares en el encinar andaluz. En el bosque caducifolio, el Carbonero Común alcanza las máximas densidades, variando sus poblaciones entre 1.9 y 13.3 parejas por $10 \mathrm{Ha}$. (véase VAN BALEN 1973). Por último, en parques y jardines urbanos, la densidad varía entre 0 y 12 parejas por $10 \mathrm{Ha}$., dependiendo del tipo de arbolado predominante (HUBLE y DHONDT 1973, ALONSO y PURROY 1969). Eliminando este último escenario, en los huertos de naranjos los valores de densidad se insertan en el campo de los obtenidos en bosques caducifolios, aunque sin alcanzar sus densidades máximas.

La disminución de la superficie arbolada no parece incidir sobre el número de parejas establecidas en el área, aunque sí lo hace sobre la distribución de las mismas, al congregarse en las zonas arboladas y abandonar aquellos huertos en los que los naranjos son arrancados. Este suceso parece indicar que, a gran escala, el efecto de la cobertura no limita directamente el número de parejas presentes en el naranjal.

En los hábitats naturales, las cavidades están limitadas (NICE 1957), y son aún más escasas en los manipulados por el hombre, al retirarse los árboles viejos y los que se secan (ALLEN y NICE 1952, BURNS 1960, VAN BALEN, 1973). La escasez de cavidades adecuadas en los naranjales puede demostrarse a partir de la utilización de la misma cavidad año tras año en determinados territorios. Así, la pareja que ocupa el territorio 1 de la figura 3, utiliza el mismo hueco en los cuatro intentos de nidificación conocidos, a pesar del fracaso de las nidadas, que son predadas sistemáticamente (dos de ellas la misma temporada). En otro hueco, el Torcecuello realizó dos puestas en 1985 y una en 1986, siendo utilizado también por una pareja de Carbonero Común, que realizó una puesta esta última temporada.

Si hay parejas que no crían, y determinadas cavidades son utilizadas año tras año, las cavidades tienen que presentar ciertos caracteres mínimos del agrado de los ocupantes, pese a que no todos tengan una «calidad» óptima. Comparando el éxito de las nidadas en relación con un determinado hueco, podemos apreciar que la cavidad utilizada por una pareja de Carbonero Común y el Torcecuello, consigue sacar adelante un total de 19 pollos (5 Carboneros y 14 Torcecuellos) en dos temporadas. Por el contrario, el hueco del territorio 1, antes descrito, no deja ningún superviviente. Las diferencias en la supervivencia entre los huecos con entradas situadas a cierta altura y los que tienen la entrada cerca del nivel del suelo, deben de guardar relación con la «calidad» de los huecos. De hecho, la cavidad utilizada por el Torcecuello y el Carbonero Común pertenece al grupo formado por nidos con aberturas situadas entre 60 y $100 \mathrm{~cm}$. del suelo, mientras que el hueco del territorio 1 se encuentra en el otro grupo.

VON HAARTMAN (1971) señala que el número de cavidades limita la población de aves que crían en ellas, aunque BRUSH (1983)sugiere que esto 
no sucede siempre. En el naranjal, los huecos limitan las parejas que consiguen nidificar, aunque la densidad es mayor.

NICE (1957) concluye que las aves nidificantes en huecos tienen un éxito reproductor mayor que las que construyen nidos abiertos. Para el Carbonero Común, en los naranjales, no es así, pues el éxito reproductor no difiere del de otras especies que crían en nidos abiertos en este mismo hábitat (véase GILDELGADO y ESCARRE 1977, GIL-DELGADO 1981). NILSSON (1975) obtiene, en huecos naturales, un éxito reproductor del $66.7 \%$, mayor que el obtenido en los naranjales, y en consonancia con las cifras propuestas por NICE (1957). Dos pueden ser las causas de esta notable diferencia. La primera estaría relacionada con la «calidad» de los huecos, ya que en el naranjal los huecos proceden exclusivamente de la putrefacción del naranjo, por lo que las condiciones que presentan para la nidificación no deben ser las adecuadas. La segunda podría estar referida a las características de los predadores potenciales. Por ejemplo, la representación de los ofidios, algunos de los cuales son conocidos predadores de huevos y pollos (VALVERDE 1967, GARZON 1974, DIAZ 1976, entre otros), es más amplia en los países mediterráneos. La anatomía de este grupo le permite introducirse fácilmente en huecos de pequeño diámetro de entrada, por lo que las especies que nidifiquen en huecos correrán mayores riesgos en áreas de coexistencia con especies de ofidios que saquean nidos. En general, las aves nidificantes en huecos en la mitad norte de Europa encontrarán en los huecos mayor seguridad que sus homólogos del sur del continente. Además, una vez localizado el nido por el predador, el hueco se convierte en una trampa para los pollos, y a veces para alguno de los padres.

En cualquier caso, la mayor seguridad que presentan los huecos resulta cuestionable en los naranajales. Cierta evidencia de la mayor presión de los predadores se desprende a partir de que, de los 8 nidos que fracasan, cinco de ellos $(62.5 \%)$ lo son por predación. Un ofidio, $M$. monspessulanus, es el predador más importante. El otro conocido, $M$. nivalis, es el principal predador de nidos de Carbonero Común en Inglaterra (DUNN 1977). La Rata Negra (Rattus rattus) también está presente en los naranjales, siendo un conocido predador de nidos de Gorrión Común (Passer domesticus) en este hábitat (GIL-DELGADO et al. 1979), y no debe despreciar las nidadas de Carbonero Común si se le presenta la ocasión.

BULMER y PERRINS (1973) y PERRINS y MOSS (1975) asumen que la mortalidad anual entre los adultos es del $50 \%$, y que la superivencia de un joven por pareja es suficiente para mantener la población estabilizada. Si observamos el sector A la figura 3D, podemos apreciar que alrededor de la mitad de las parejas no logra nidificar. BARBA (1986) señala que, en los naranjales, sólo el $9 \%$ de las parejas hace segundas puestas y, entre los que intentan criar, la producción es del 1.7 pollos por nido (tabla I), valor que se reduce si relacionamos la producción de pollos con el número de parejas asentadas en la parcela. BULMER y PERRINS (1973) señalan que el $78 \%$ de los jóvenes mueren durante el primer año de vida, después de abandonar el nido. En los naranjales, este valor debe de ser inferior, al ejercer un mayor peso la mortalidad durante la fase de estancia en el nido. 


\section{BIBLIOGRAFIA}

ALLEN, R. W. y NICE, M. M. 1952: A study of the breeding biology of the Purple Martin (Progne subis). Am Midl. Nat. 47: 606-665.

ALONSO, J. A. y PURROY, F. J. 1979: La avifauna de los parques de Madrid. Naturalia hispanica 18.

BARBA, E. 1986: El Carbonero Común (Parus major) en el naranjal. Tesis de Licenciatura no publicada.

BLONDEL, J. 1965: Etude des populations d'oiseaux dans une garrigue méditerrannée: description du milieu, de la méthode de travail et exposé des premiers résultats obtenus á la période de reproduction. La Terre et La Vie 112: 331-341.

BRUSH, T. 1983: Cavity use by secondary cavity-nesting birds and response to manipulations. Condor 85: 461-466.

BULMERR, M. G. y PERRINS, C. M. 1973: Mortality in the Great Tit Parus major. Ibis 115: 277-281.

BURNS, H. 1960: The economic importance of birds in forests. Bird Study 7: 193-208.

DIAZ, C. 1976: Alimentación de la Culebra Bastarda (Malpolon monspessulanus; Ophidia, Colubridae) en el S. O. de España. Doñana, Acta Vertebrata 3: 113-127.

DUNN, P. K. 1977: Predation by Weasels (Mustela nivalis) on breeding tits (Parus ssp.) in relation to the density of tits and rodents. J. Anim. Ecol. 46: 633-652.

ESCOBAR, J. V. y GIL-DELGADO, J. A. 1984: Estrategias de nidificación en Passer domesticus. Doñana, Acta Vertebrata 11 (1): 65-78.

GARCIA, L. y PURROY, F. J. 1973: Evaluaciones de comunidades de aves por el método de la parcela. Resultados obtenidos en el matorral mediterráneo de la Punta del Sabinar, Almería. Bol. est. cen. ecol. 2: 41-49.

GARZON, J. 1974: Coluber hippocrepis acechando Hirundo rustica y capturando Passer domesticus. Doñana, Acta Vertebrata 1: 51.

GIL-DELGADO, J. A. 1981: La avifauna del naranjal valenciano. III. El Verdecillo (Serinus serinus L.). Mediterránea 5: 97-114.

- 1983: Breeding bird community in orange groves. Proc. VII Int. Con. Bird Census IBCC $V$ Meeting EOAC, 100-106.

GIL-DELGADO, J. A. y ESCARRE, A. 1977: Avifauna del naranjal valenciano, I. Datos preliminares sobre el Mirlo (Turdus merula L.). Mediterránea 2: 89-109.

GIL-DELGADO, J. A.; PARDO, R.; BELLOT, J. y LUCAS, T. 1979: Avifauna del naranjal valenciano. II. El Gorrión Común (Passer domesticus L.) Mediterránea 3: 69-99.

HAARTMAN, L. Von 1971: Population dynamics. En Avian Ecology, vol. I. pp. 391-459. Farner D. S. y King J. R. (eds.) Academic Press, New York.

HERRERA, C. M. 1978: Evolución estacional de las comunidades de Passeriformes en dos encinares de Andalucía Oriental. Ardeola 25: 143-180.

HUBLE, J. y DHONDT, A. A. 1973: Ecologie des populations de Mésanges Charbonniéres ( $P a-$ rus m. major L.) en basse Belgique. Extrait des Ann. Soc. Royale Zool. de Belgique 103 (1): $37-41$.

KREBS, J. R. 1971: Territory and breeding density in the Great Tit, Parus major L. Ecology 52: 2-22.

LACK, D. 1958: A quantitative breeding study of British tits. Ardea 47: 91-124.

NICE, M. M. 1957: Nesting success in altricial birds. $A u k$ 74: 305-321.

NILSSON, S. G. 1975: Kullstorlek och hckningsframgang in holkar och naturliga hal. Var Fagelvarld 34: 207-211.

PEDROCHI, C. 1973: Estudios en bosques de coníferas del Pirineo Central. Serie A: pinar con acebo de San Juan de la Peña. 2) «Utilización de métodos de cuadrícula al estudio de la densidad de nidificación de aves». Pirineos 109: 73-77.

- 1975: Efecto topoclimático en la densidad de nidificación de aves. P. Cent. pir. Biol. exp. de Jaca 7: 163-167. 
PERRINS, C. M. 1979: British Tits. Collins, London.

PERRINS, C. M. y MOSS, D. 1975: Reproductive rates in Great Tit. J. Anim. Ecol. 44: 695-706.

SMITH, H. M. 1943: Size of breeding populations in relation to egg laying and reproductive success in the Eastern Red Winged Blackbird (Agelaius phoeniceus). Ecology 24: 138-207.

VAL NOLAN Jr. 1963: Reproductive success of birds in decidous scrub habitat. Ecology 44: 305-313.

VALVERDE, J. A. 1967: Estructura de una comunidad de vertebrados terrestres. C.S.I.C., Madrid. 